\title{
Volker Matthies: Im Schatten der Entdecker. Indigene Begleiter europäischer Forschungsreisender
}

Berlin: Chr. Links Verlag 2018 - ISBN 978-3-86153-989-6 - 28,00€

»Alexander von Humboldt erforschte Südamerika - hat er den Weg allein gefunden? « Diese Anspielung auf Brechts Fragen eines lesenden Arbeiters (1935) steht auf der Umschlagrückseite des Buchs Im Schatten der Entdecker von Volker Matthies. Schlägt man den Band auf, sieht man dann vier Zeilen von Brechts Gedicht, die ihm als Motto vorangestellt sind. Die Programmatik dürfte damit deutlich sein: Es geht darum, die Geschichte der Entdeckungen vom Kopf auf die Füße zu stellen, und zwar ganz buchstäblich: Klappt man das Buch noch einmal zu, dann sieht man als Umschlagbild den britischen Afrikaforscher Verney Lovett Cameron, wie er, im Stuhl sitzend, auf dem Rücken eines indigenen Trägers den Lulundi überquert. Wie aber heißt der Träger? Hier beginnen die Fragen, mit denen sich dieses Buch beschäftigt, das erklärtermaßen einen Beitrag zur »Entkolonisierung und Entheroisierung« (9) der Entdeckungsgeschichte sein will. Das Ergebnis ist sehr lesenswert.

Die Geschichte der europäischen Entdeckungsunternehmungen vom 15. bis ins 20. Jahrhundert ist auch immer eine Geschichte der Zusammenarbeit von Europäern und Nichteuropäern (die freilich sehr unterschiedliche Züge annehmen kann). Matthies spricht daher von »kollaborative[n] Unternehmen« bzw. »gemeinsame[n] Projekt[en]« (23) von Europäern und Indigenen. Dennoch ist sie kanonisiert als die Geschichte bzw. der »Abenteuerroman« (19) männlicher europäischer Entdecker und wird bis heute unter deren Namen allein tradiert. Die Namen der Anderen sind auch gar nicht mehr festzustellen, wenn sie nicht innerhalb der europäischen Zeugnisse dieser Entdeckungsreisen erhalten sind.

Wenn Matthies aber schreibt, es lasse »sich selbst mit den vergleichsweise eher spärlichen Informationen ein beeindruckendes Bild dieser Menschen zeichnen« (25), so ist das im Ergebnis völlig zutreffend. Man kann hinzufügen: Dieser Band demonstriert die Zeugnisse über indigene Begleiter und insbesondere das Verhältnis zwischen europäischen und nichteuropäischen Expeditionsteilnehmern in solcher Dichte, dass der ursprüngliche Befund eines Informationsmangels gar nicht mehr auffällt und tatsächlich eigens erwähnt werden muss. Der Band geht dabei auf viele einschlägige Forschungen anderer zurück, die er zu einer facettenreichen Darstellung bündelt. Er bietet nicht nur Fallgeschichten, sondern gleich im ersten Hauptteil (»Indigene Begleiter - die Leistungen«, 27-114) einen breiten historischen Überblick, der freilich immer eng geknüpft an das historisch Konkrete und das ausgewertete Material bleibt.

So tritt man ein in die Ambivalenzen, die mit der Kooperation zwischen europäischen >Entdeckern < und indigenen >Begleitern verbunden sind. Auch wenn die Kooperation oftmals mit Gewalt und Ausbeutung einhergeht, sind die Machtverhältnisse auf 
Entdeckungsreisen dabei selten eindeutig. Der englische Afrikaforscher Joseph Thomson etwa, der als junger Mann mit den Erfordernissen einer Expedition in Ostafrika selbst eher überfordert ist, gilt den indigenen Expeditionsteilnehmern als der »white man« (69) des indigenen Expeditionsführers Chuma. Auch wenn Thomson dieses Verhältnis in seiner eigenen Darstellung Through Masai Land (1885) nicht verhehlt, spricht die Ikonographie darin schon eine andere Sprache. In Tropenuniform sitzt da der europäische Entdecker, den Kopf in die Hand gestützt, umgeben von zwei barfüßig stehenden Begleitern, einer von ihnen ebenjener Chuma.

Tatsächlich sind europäische Entdeckungsreisende in vielen Fällen lokalen Machthabern ausgeliefert und müssen Wege finden, sich mit ihnen $\mathrm{zu}$ arrangieren. Irgendeine Art der Konvergenz zwischen europäischen Interessen und denen lokaler Autoritäten gibt es fast immer, sei es Machtzuwachs oder (Sklaven-)Handel, und manche Expedition verdankt ihr Zustandekommen gar deren Initiative. Die Nilquellen-Expedition der Briten Burton und Speke findet unter der Flagge des Sultanats Oman-Sansibar statt. Oftmals sind die europäischen Entdecker ohne die Kenntnisse indigener Expeditionsteilnehmer - und ganz ausdrücklich: Expeditionsteilnehmerinnen - in den unvertrauten Regionen schlichtweg nicht überlebensfähig. Erst deren Kleidung, Nahrungsmittel, geographische Kenntnisse, aber auch sprachliche und diplomatisch-kulturelle Fähigkeiten bewahren die Expeditionen vor dem Scheitern.

Der zweite Teil (»Indigene Begleiter -ausgewählte Biographien«,115-183) fügt dem Buch nicht allein anschauliche Einzelbiographien - und damit die Namen der Indigenen als Subjekte in den Überschriften - hinzu, sondern darin eingeschlossen auch die dramatische Kehrseite der im ersten Teil beschriebenen wesentlichen Partizipation Indigener an den Leistungen seuropäischer Forschungsreisen. Denn diese Partizipation ist, erstens, oftmals mit der Aufgabe soziokultureller Identität verbunden (Matthies greift auf den Begriff der >deracination $<$ des Historikers Dane Kennedy zurück, vgl. 186), was im konkreten biographischen Schicksal $\mathrm{zu}$ durchaus tragischen Konsequenzen führt. Matonabbee, der indianische, aber in einem Fort der Hudson's Bay Company großgezogene Führer (und das heißt eben auch Anführer) der Hearne'schen Kanada-Expedition, bringt sich um, als die Franzosen das britische Fort zerstören und sich mit der Machtverschiebung auch seine Stellung in der indianischen Gesellschaft von einer privilegierten zu einer prekären Außenseiterschaft wandelt. Die Schoschonin Sacagawea und schon die mexikanische Indianerin Malinche (beide schon früh durch Versklavung ihren indianischen Herkunftsgesellschaften entrissen) helfen den Entdeckern bzw. Eroberern mit ihren Orts- und vor allem Sprachkenntnissen erheblich. Damit tragen sie, zweitens, zur Ausbeutung und Zerstörung ihrer Herkunftsgesellschaften und -länder bei.

In einem also weit über die persönlichen Schicksale hinausgehenden Sinn läuft dies auf die Frage hinaus, die Matthies am Ende des Buchs stellt: Waren die indigenen Begleiter »Wegbereiter des europäischen Impe- 
rialismus« (185)? Die Antwort fällt ambivalent aus. Selbstverständlich sind die Entdeckungsunternehmen mit ihrem erheblichen finanziellen und logistischen Aufwand Teil der globalen europäischen Expansion. Indem sie Landkarten erstellen oder Handelswege und Posten etablieren helfen, leisten die indigenen Begleiter einen wesentlichen Beitrag dazu. Ihre Motive sind aber biographischen und lokalen Begrenzungen unterworfen. Wie man schwerlich Alexander von Humboldts Südamerika-Reise, die ihren Erfolg auch den imperialistischen Inter- essen Spaniens verdankt, insgesamt als imperialistisches Unternehmen wird abtun können, so wird man auch die manchmal aus der Not geborenen, manchmal kalkulierten Entscheidungen Indigener, sich an den Expeditionen zu beteiligen, nicht allein im Licht ihres Nutzens für den europäischen Imperialismus sehen können. Im Gegenteil, die Erforschung ihrer Leistungen ist eine Aufgabe, die auch zur Würdigung ihrer komplizierten Geschichte beiträgt.

Hans-Christian Riechers

\section{Esaïe Djomo (Hg.): Lieder der alten Afrikaner. Anthologie deutschsprachiger Koloniallyrik 1884-1945. Mit einem Vorwort v. Esaïe Djomo}

St. Ingbert: Röhrig 2017 - ISBN 978-3-86110-631-9 - 29,00€

Im Jahr 1992 ist bei Röhrig als 31. Band der Saarbrücker Beiträge zur Literaturwissenschaft die Dissertation Esaïe Djomos mit dem Titel »Des Deutschen Feld, es ist die Welt!« Pangermanismus in der Literatur des Kaiserreichs, dargestellt am Beispiel der deutschen Koloniallyrik. Ein Beitrag zur Literatur im historischen Kontext erschienen. Ein Vierteljahrhundert später legt der Verfasser nun im selben Verlag eine Anthologie vor, deren Umfang im Wesentlichen dem damals untersuchten Textkorpus entspricht, wie das Vorwort der Lieder der alten Afrikaner eingangs auch sogleich betont (vgl. 17). Insofern scheint es durchaus angemessen, zunächst einige Worte über die Dissertation von $1992 \mathrm{zu}$ verlieren, zumal deren Konzeption die Auswahl der Texte auch für die Anthologie von 2017 offensichtlich entscheidend bestimmt.
Wie bereits der Untertitel der älteren Untersuchung hervorhebt, besteht deren Bedeutung in erster Linie in der Einbettung des kolonialen Diskurses in die Gedankenwelt des Pangermanismus, der vom späten Kaiserreich bis zum Beginn des Dritten Reiches stetig an Einfluss gewinnt. Dazu weist Djomo in den untersuchten Texten eine Typologie der Themen nach, die den pangermanistischen Kolonialdiskurs in besonderem Maße kennzeichnen, wie die Lebensraumfrage, die Kriegsbegeisterung, die Flottenpolitik oder die Germanisierung der Kolonien. Als dominantes stilistisches Mittel der untersuchten Koloniallyrik gilt Djomo die Kontrafaktur, die sich insbesondere in der Form äußert, dass zahlreiche Gedichte nach Strophenform und Melodie bekannter patriotischer Gesänge oder populärer Volkslieder verfasst werden. Neben 